\title{
Comparison of methods and co-registration maps of EEG and fMRI in Occipital Lobe Epilepsy
}

\author{
M. Forjaz Secca ${ }^{1,2}$, A. Leal ${ }^{3,4}$, J. Cabral $^{1}$ and H. Fernandes ${ }^{1}$ \\ ${ }^{1}$ Cefitec, Dep. of Physics, Universidade Nova de Lisboa, Portugal \\ ${ }^{2}$ Ressonância Magnética de Caselas, Lisboa, Portugal \\ ${ }^{3}$ Department of Neurophysiology, Hospital Júlio de Matos, Lisboa, Portugal \\ ${ }^{4}$ Department of Pediatric Neurology, Hospital Dona Estefânia, Lisboa, Portugal
}

\begin{abstract}
Clinically childhood occipital lobe epilepsy (OLE) manifests itself with distinct syndromes. The traditional EEG recordings have not been able to overcome the difficulty in correlating the ictal clinical symptoms to the onset in particular areas of the occipital lobes. To understand these syndromes it is important to map with more precision the epileptogenic cortical regions in OLE. Experimentally, we studied three idiopathic childhood OLE patients with EEG source analysis and with the simultaneous acquisition of EEG and fMRI, to map the BOLD effect associated with EEG spikes. The spatial overlap between the EEG and BOLD results was not very good, but the fMRI suggested localizations more consistent with the ictal clinical manifestations of each type of epileptic syndrome. Since our first results show that by associating the BOLD effect with interictal spikes the epileptogenic areas are mapped to localizations different from those calculated from EEG sources and that by using different EEG/fMRI processing methods our results differ to some extent, it is very important to compare the different methods of processing the localization of activation and develop a good methodology for obtaining co-registration maps of high resolution EEG with BOLD localizations.
\end{abstract}

Keywords- fMRI, BOLD, EEG, Epilepsy.

\section{INTRODUCTION}

EEG is useful in localizing epileptic activity to the occipital lobes in childhood occipital lobe epilepsies (OLEs), however OLE manifests itself with different syndromes and EEG localization is not syndrome-specific with abnormalities very often also involving the parietal and temporal areas [1]. The topography of spikes rarely points to the particular region in the occipital lobes originating the seizures and the few attempts to do source analysis did not improve the generally poor electroclinical correlation [2]. In the case of idiopathic epilepsies, where brain imaging is normal, the particular regions of seizure onset remain unknown, despite the consistent clinical picture of each syndrome. The detection of the BOLD effect associated with the occurrence of interictal spikes in simultaneous EEG/fMRI recordings offers a promising way to detect the epileptic neuronal dysfunction with high spatial resolution [3]. Some studies with this method have been done in symptomatic OLE [3-5] demonstrating significant posterior activation. No work has been done in idiopathic OLE, a group of epilepsies where clues to the particular cortical area of onset are lacking. The goals of this work are to use the BOLD effect associated with the occurrence of interictal spike activity in different types of childhood OLE to improve the electroclinical correlation and also to compare the degree of concordance of this technique with the conventional methods of EEG source analysis.

\section{MATERIALS AND METHODS}

We studied three patients with a diagnosis of idiopathic OLE, submitting them to a 60-min EEG recording outside the scanner, including a sleep period, with a cap of $36 \mathrm{AgCl}$ electrodes (Fp1/2, F3/4, FC3/4, C3/4, CP3/4, P3/4, PO1/2, O1/2, F7/8, FT7/8, T3/4, TP7/8, T5/6, FT9/10, A1/2, Fz, $\mathrm{FCz}, \mathrm{Cz}, \mathrm{CPz}, \mathrm{Pz}, \mathrm{Oz}$ ). We used a sampling rate of $256 \mathrm{~Hz}$, filters of $0.5-70 \mathrm{~Hz}$, and performed intermittent photic stimulation at the end of registration. Later in the same day, a session of functional MRI was performed while simultaneously recording the EEG (19 electrodes at standard 10-20 positions). Each patient demonstrated a single, topographically stable, paroxysm type. This was the main neurophysiological criteria for selection of patients for this study. Informed consent was obtained from the parents of the patients.

Source analysis of the EEG was done in spikes detected visually in recordings obtained outside the scanner in all patients and also inside for patient 1. The EEG was high pass filtered at $3 \mathrm{~Hz}$ (zero phase shift filter with $24 \mathrm{~dB} / \mathrm{oct}$ ) and spikes with good signal to noise ratio were aligned by the peak amplitude to produce an average spike. For patients 2 and 3, not enough spikes could be recorded inside the scanner to produce stable dipole solutions. The sources were obtained from instantaneous moving dipoles at the peak of averaged spikes ( $n=43,19$, and 21 for patients 1,2, and 3), with a standard three layer Boundary Element Model (BEM) volume conductor model (conductances of 
0.33, 0.0042, $0.33 \mathrm{~S} / \mathrm{m}$ for scalp, bone and brain), provided in the Source2 software package (Neuroscan, El Paso, Mexico). Standard electrode positions were also used. The confidence ellipsoids for the dipole positions represent the $1 \mathrm{SD}$ interval and are directly proportional to the noise estimate in the averaged spikes [6], evaluated as $5 \mu \mathrm{V}, 6 \mu \mathrm{V}$, and $5 \mu \mathrm{V}$, for patients 1,2 , and 3 .

The EEG/fMRI consisted in the acquisition of blocks of 100 brain volumes each one made of 16 EPI images (in plane resolution $3.75 \mathrm{~mm}$ and slice thickness of $7 \mathrm{~mm}$, no spacing; echo time $50 \mu \mathrm{s}$; flip angle of $90^{\circ}$ ) obtained with a $\mathrm{TR}=3 \mathrm{~s}$, corresponding to periods of $5 \mathrm{~min}$ of continuous and simultaneous monitoring. 4 to 6 blocks were obtained per patient, providing 20 min of simultaneous monitoring for patient 1,30 min for patient 2, and $20 \mathrm{~min}$ for patient 3 . A brain $\mathrm{T} 1$ weighted anatomic sequence (in plane resolution $0.94 \mathrm{~mm}$ and slice thickness of $1.3 \mathrm{~mm}$ ) was obtained in the same session.

Images were acquired in a $1.5 \mathrm{~T} \mathrm{GE} \mathrm{CVi} / \mathrm{NVi}$ scanner, while the EEG was recorded through a set of $\mathrm{AgCl}$ electrodes connected to an amplifier located outside the scanner room through carbon fiber wires (MagLink, Neuroscan, El Paso, TX, U.S.A.). The cap did not produce detectable artifacts in the MRI sequences, so these could be processed without any special correction. The EEG was corrected for artifacts induced by the magnetic field and rapidly changing imaging gradients offline using commercial software (Scan 4.3.2, Neuroscan). The time of occurrence of spikes was determined by visual inspection and used to classify the acquired image volumes, resulting in sequences of events of interest used to build an eventrelated paradigm. To model the hemodynamic function a standard Gamma function with derivatives was used [7]. Sixty, three and nine spikes were analyzed, respectively, for patients 1, 2, and 3. The EPI sequences were corrected for movement and slice acquisition time and smoothed with a Gaussian kernel of FWHM $8 \mathrm{~mm}$. A local autocorrelation correction was used [8] and $\mathrm{z}$ statistic images generated. The correction for the multiple comparison problem was done using a cluster threshold with $p=$ 0.05 . The preprocessing and paradigm-related analysis of the fMRI was performed using the FSL software package [9].

Representation of dipoles on individual brain anatomy was performed by adjusting the fiducial points (nasion, preauricular points, inion, and vertex) of the BEM model on the individual 3D T1 MRI. Representation on the inflated cortex was done with the FreeSurfer software package [10].

\section{RESULTS}

The recognition of interictal spikes was possible with the EEGs obtained inside the scanner (Figs. 1C and 2C). The number of these spikes was clearly reduced when compared to the recordings outside the scanner.

We present here the results of two of our patients, as an example.

For patient 1 the EEG background is normal and high amplitude spikes are present over the right occipitalparietal region (Fig. 1A), most often occurring rhythmically at 1-3 $\mathrm{Hz}$ and with a clear reduction while recording with the eyes open. The spike morphology and topography are monotonous and demonstrate a clear bipolar scalp distribution of the electrical potential (Fig. 1B). The source analysis reveals dipoles located over the cortical right parietal-occipital region (Fig. 1B), suggesting a restricted focal epileptogenic area. The source obtained from EEG inside the scanner shows a similar localization but suffers from worse signal to noise ratio (larger confidence ellipsoid) and deficient spatial sampling over right temporal areas (solution shifted to the left) (Fig. 1C). The BOLD activation associated with the occurrence of spikes involves the more posterior and medial areas of both parietal lobes, with small clusters in the neighborhood of the calcarine fissure (Figs. 1D,E). No significant BOLD activation is apparent in the cortex adjacent to the source analysis dipoles. Deactivation analysis produces several clusters, with a more consistent one over the right parietal area, in a localization similar to that of EEG source analysis.

Patient 3 presents spikes and sharp waves with maximum amplitude over occipital electrodes (Fig. 2A). The average paroxysm shows a dipolar potential field over the scalp, and the dipole solution locates the generators in the medial occipital lobe (Fig. 2B), but the spatial stability of the solution is low (large confidence ellipsoid) due to the poor signal to noise ratio. The BOLD activations are located over the posterior and medial occipital cortex and also over the basal ganglia in the right hemisphere (Figs. 2D,E). The ones localized in the occipital lobe are more superficial than the dipole solutions, and point to different cortical areas as the source of scalp paroxysms. The BOLD deactivation occurs in the left frontal lobe.

\section{Conclusions}

We observed a clear discrepancy between the localization of epileptogenic cortical areas suggested by the EEG source analysis and the BOLD activations associated with the paroxysms. This occurs despite the fact that the spatial sampling of the EEG over posterior areas was increased [2] resulting in five electrodes over occipital areas (as compared with two for the 10-20 system) and an even more significant increase over parietal and temporal areas (Figs. $1 \mathrm{~B}$ and $2 \mathrm{~B})$. 
a)

$\mathrm{Fp} 2$

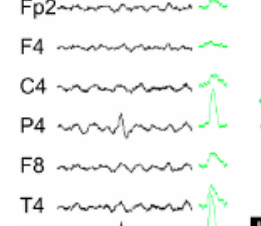

T6 2

Fp1 1 mann

F3

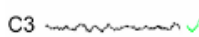

P3 nuniming

F7

T3 mancinams

T5 mann

01 ambirma

$\mathrm{Fz}$

$\mathrm{Cz}$

$\mathrm{Pz}$ unveram

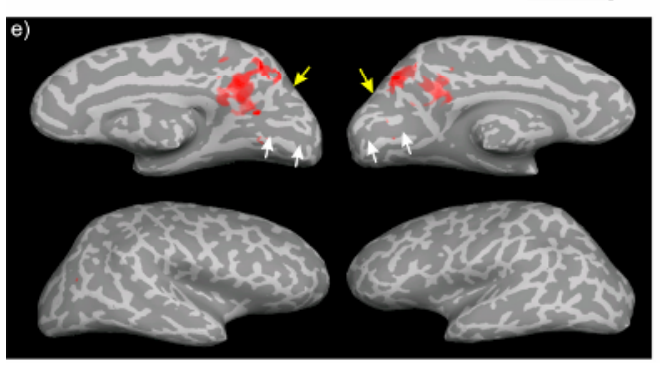

c)
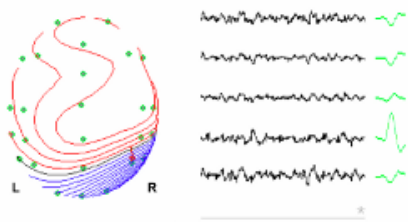

trimpromentions
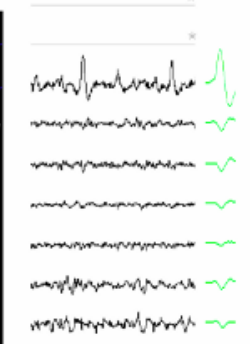

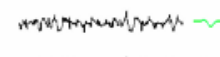

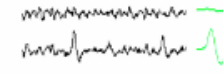

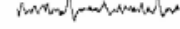

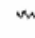

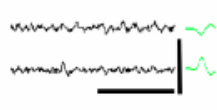

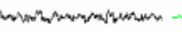
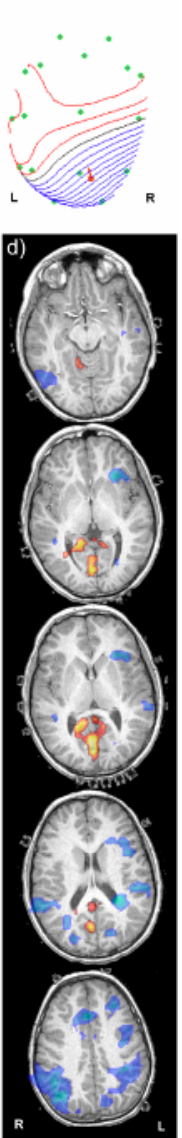

Fig. 1 (A) Sample of selected EEG channels demonstrating the interictal spikes in patient 1 over the posterior right hemisphere (the scale bar represents $1 \mathrm{~s}$ of time horizontally and $200 \mu \mathrm{V}$ vertically) and average spike at right $(n=43)$. Above right, map of electrical potential at the peak of an averaged spike (electrodes are green, nose upward; negative potentials are blue and positive ones red). (B) Moving dipolesolution around the peak of an averaged spike outside (red) and inside (blue) the scanner, with a measure of dispersion represented in confidence ellipsoids (1 SD); (C) Raw spikes inside the scanner after artifact correction, with average $(\mathrm{n}=15)$ and potential map on the scalp ( $\square$ represents bad channels); (D) BOLD regions of significant activation (red) and deactivation (blue) represented over the patient high resolution T1 MRI; (E) Activation regions represented on the inflated white/gray matter interface. The calcarine and parietaloccipital sulci are indicated by white and yellow arrows, respectively.

The fact that the EEG used for source analysis was obtained outside the scanner room evokes the possibility that this discrepancy might be due to different paroxysm types being analyzed in the two methods. This is unlikely because patients were selected to the study with the requirement that they had topographically stable, single paroxysm types in previous EEGs; the data collection was done in the same day; and the paroxysms in the EEG obtained inside and outside the scanner room showed a similar topography (Figs. 1A-C and 2A-C). Dipole localization was clearly a)

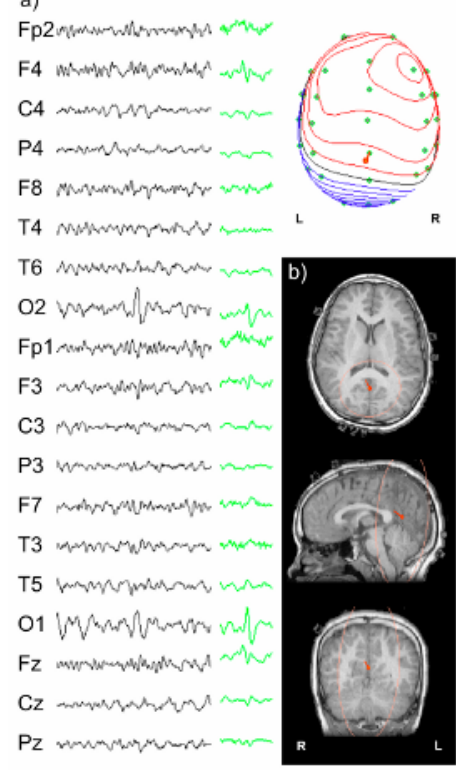

c)
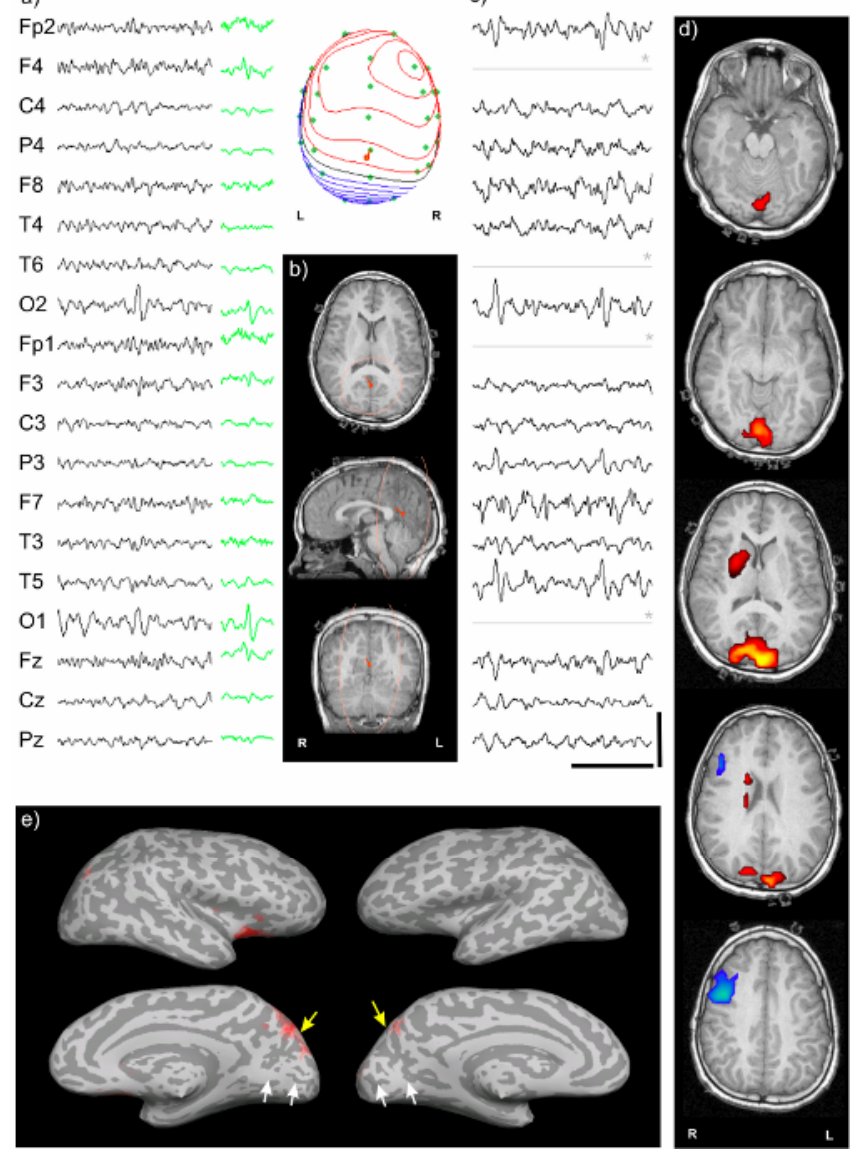

Fig. 2 (A) Sample of EEG with posterior paroxysms over the posterior electrodes. Right, average spike $(n=21)$; (B) Scalp potential map with bipolar distribution at the peak of an average spike. Below, dipole solution at the spike peak located near the primary visual areas, but with a large error ellipsoid associated; (C) Raw EEG inside the scanner with posterior spikes; (D) BOLD activation (red) associated with the spikes, in the posterior areas of both hemispheres and in the basal ganglia on the right. Deactivation over the frontal lobe is shown in blue; (E) Activation clusters over the parietaloccipital region. The activation in the lower right frontal lobe results from artifactual projection of the basal ganglia activation on the nearest surface (scale bars, colors, and arrows as in Fig.1).

discrepant in relation to BOLD activations, while there was concordance with the largest cluster of deactivation.

The overall concordance of BOLD deactivation with the source analysis is no better than for activation, a point already mentioned in the literature [12]. We could obtain BOLD activations in $100 \%$ of our patients, which is significantly better than the results of larger series, were values in the order of $40-60 \%$ are mentioned $[3,13]$ for patients with focal epilepsy. A study in a population with generalized idiopathic epilepsy [11] improves this value to $93 \%$, and together with our results suggest that the method may be 
more efficient in idiopathic epilepsies as compared with the symptomatic ones. Overall, our data show different BOLD activations on the occipital lobes in different syndromes of idiopathic OLE. The epileptogenic regions identified by this method show important discrepancies with the ones suggested by dipole analysis, in line with data from the literature $[12,14]$, but as a possible representation of the epileptogenic area, they provide a more satisfactory explanation of the clinical ictal manifestations from the literature.

Our first clinical results have already been published [15], but since our experimental analysis also shows that by using different EEG/fMRI processing methods our results differ to some extent, it is very important to compare the different methods of processing the localization of activation and develop a good methodology for obtaining coregistration maps of high resolution EEG with BOLD localizations, which is what we are working on at present.

The EEG/fMRI technique is a powerful method for the study of occipital lobe epilepsies and can provide a way to build more detailed models integrating clinical, structural, electrical, and vascular data.

\section{ACKNOWLEDGMENT}

The authors would like to thank Cristina Menezes, Elisa Vilar, Rita Pinto, Elizabete Lage, and Bruno Mourão for their technical support. The work was supported by a grant for Research in Epilepsy from Tecnifar SA and by projects Topo3D (POSI/CPS/39758/2001) and EpilBI (POSC/EEACPS/60977/2004) from FCT.

\section{REFERENCES}

1. Taylor I, Scheffer I, Berkovic S. Occipital epilepsies: identification of specific and newly recognized syndromes. Brain 2003;126:753-69.

2. Van der Meij W, Van der Dussen, Huffelen V, et al. Dipole source analysis may differenciate benign focal epilepsy of childhood with occipital paroxysms from symptomatic occipital lobe epilepsy. Brain Topogr 1997;10:115-20.

3. Al-Asmi A, B'enar C, Gross D, et al. fMRI activation in continuous and spike-triggered EEG-fMRI studies of epileptic spikes. Epilepsia 2003;44:1328-39.
4. B'enar C, Gross D, Wang $\mathrm{Y}$, et al. The BOLD response to interictal epileptiform discharges. Neuroimage 2002; 17:1182-92.

5. Lazeyras F, BlankeO, Perrig S, et al. EEG-triggered functional MRI in patients with pharmacoresistant epilepsy. J Mag Res Imaging 2000;12:177-85.

6. Fuchs M, Wagner M, Kastner J. Confidence limits of dipole source reconstruction results. Clin Neurophysiol 2004; 115:1442-51.

7. Huettel S, McKeown J, Song A, et al. Linking hemodynamic and electrophysiological measures of brain activity: evidence from functional MRI and intracranial field potentials. Cereb Cortex 2004;4:165-73.

8. Worsley KJ, EvansAC, Marrett S, Neelin P.Athree-dimensional statistical analysis for CBF activation studies in human brain. J Cerebr Blood Flow and Metab 1992;12:900-18.

9. Smith S, Jenkinson M, Woolrich M, et al. Advances in functional and structural MR image analysis and implementation as FSL. Neuroimage 2004;23(suppl 1):208-19.

10. Fischl Bruce, Sereno M.I., Dale A.M. Cortical surface-based analysis II: inflation, flattening, and a surface-based coordinate system. Neuroimage 1999;9:195-207.

11. Aghakhani Y, Bagshaw AP, B'enar CG, et al. fMRI activation during spike and wave discharges in idiopathic epilepsy. Brain 2004;127:1127-44.

12. BagshawA,Kobayashi E, Dubeau F, et al. Correspondence between EEG-fMRI and EEG dipole localization of interictal discharges in focal epilepsy. Neuroimage 2006;30:417-25.

13. Bagshaw A, Aghakhani Y, B'enar C, et al. EEG-fMRI of focal epileptic spikes: analysis with multiple haemodynamic functions and comparison with Gadolinium-enhanced MR angiograms. Hum Brain Mapp 2004;22:179-92.

14. Lemieux L, Krakow K, Fish DR. Comparison of spiketriggered functional MRI BOLD activation and EEG dipole model localization. Neuroimage 2001;14:1097-104.

15. Leal A, Dias A, Vieira JP, Secca M, Jordao C. The BOLD effect of interictal spike activity in childhood occipital lobe epilepsy. Epilepsia. 2006 Sep;47(9):1536-42.

Address of the corresponding author:

Author: Mario Forjaz Secca

Institute: Cefitec, Physics Department, Universidade Nova de Lisboa

Street: Quinta da Torre

City: $\quad 2829-516$ Caparica

Country: Portugal

Email: mfs@fct.unl.pt 